\title{
Is end-stage lateral osteoarthritic knee always valgus? Mechanical alignment analysis and radiographic severity assessment
}

\author{
Su Chan Lee ${ }^{1}$ Viral Gondalia ${ }^{1}$ Byoung Yoon Hwang ${ }^{1} \cdot$ Hye Sun Ahn ${ }^{1}$. \\ Choon Key Lee ${ }^{1} \cdot$ David J. Hunter ${ }^{2} \cdot$ Kwang Am Jung ${ }^{1}$
}

Received: 16 April 2014/ Accepted: 15 May 2015/Published online: 3 June 2015

(C) The Author(s) 2015. This article is published with open access at Springerlink.com

\begin{abstract}
Background We hypothesized that not all persons with end-stage lateral osteoarthritis (OA) have valgus malalignment and that full extension radiographs may underreport radiographic disease severity. The purpose of this study was to examine the demographic and radiographic features of end-stage lateral compartment knee OA.

Materials and methods We retrospectively studied 133 knees in 113 patients who had undergone total knee arthroplasty between June 2008 and August 2010. All patients had predominantly lateral idiopathic compartment OA according to the compartment-specific KellgrenLawrence grade (KLG). The mechanical axis angle (MAA), compartment-specific KLG and joint space narrowing (JSN) of the tibiofemoral joint at extension and $30^{\circ}$ of knee flexion, tibia vara angle, tibial slope angle, body mass index, age, and sex were surveyed.

Results End-stage lateral compartment knee $\mathrm{OA}$ has varus (37.6\%), neutral (22.6\%), and valgus (39.8\%) MAA on both-leg standing hip-knee-ankle radiographs. KLGs at $30^{\circ}$ of knee flexion (fKLG) were grades 3 and 4 in all patients. However, for KLGs at full extension (eKLG), $54 \%$ of all patients had grades 3 and 4 . The others (46\%) showed grades 1 and 2 . We observed significant
\end{abstract}

Kwang Am Jung

kwangamj@gmail.com

1 Joint and Arthritis Research, Department of Orthopaedic Surgery, Himchan Hospital, 20-8, Songpa-dong, Songpa-gu, Seoul 138-170, Korea

2 Rheumatology Department, Royal North Shore Hospital and Northern Clinical School, University of Sydney, Sydney, NSW, Australia differences in lateral compartment eKLG/eJSN (2.3/ $2.3 \mathrm{~mm}$ in varus, $2.5 / 1.9 \mathrm{~mm}$ in neutral, $2.9 / 1.6 \mathrm{~mm}$ in valgus, $p=0.01$ and 0.03 , respectively), tibia vara angle $\left(4.9^{\circ}\right.$ in varus, $4.1^{\circ}$ in neutral, $3.0^{\circ}$ in valgus, $\left.p<0.01\right)$, and medial compartment eKLG/eJSN $(2.1 / 3.1 \mathrm{~mm}$ in varus, $2.0 / 3.4 \mathrm{~mm}$ in neutral, $1.8 / 4.3 \mathrm{~mm}$ in valgus, $p<0.01$ and 0.01 , respectively) between MAA groups, except for the tibial slope angle $\left(9.7^{\circ}\right.$ in varus, $10.1^{\circ}$ in neutral, $9.8^{\circ}$ in valgus, $p=0.31$ ).

Conclusion Varus alignment was paradoxically shown in approximately one-third of those with end-stage lateral knee OA on both-leg standing hip-knee-ankle radiographs. Films taken in full extension underreported the degree of OA radiographic severity.

Level of evidence Level IV, observational study.

Keywords Lateral compartment - Osteoarthritis - Knee

\section{Introduction}

Osteoarthritis (OA) in the knee joint is the most common disorder in orthopedics and is characterized by structural and functional failure of the synovial joint tissue with loss and erosion of articular cartilage, subchondral bone alteration, meniscal degeneration, and bone and cartilage erosion [1].

Conventional radiography is the most convenient and important imaging examination in a clinical setting when evaluating a patient who has a known or suspected diagnosis of OA. Radiographs clearly visualize bony features, including marginal osteophytes, subchondral sclerosis, and bone cysts, but provide only an estimate of cartilage thickness and meniscal integrity by joint space narrowing (JSN). However, progression of JSN is the most commonly 
used criterion for the assessment of OA progression, and the complete loss of joint space width, characterized by interbone contact is one of the factors considered in the decision for joint replacement.

Radiography is an indispensable complement to clinical examination and plays a key role in diagnosing and monitoring the course of a condition in OA. Lower limb alignment and extended and/or semi-flexed knee anteroposterior radiographs can be used for evaluating the relationship between $\mathrm{OA}$ and compartmental pattern and severity of knee OA.

However, most studies have focused on patients with early stage OA. In addition, there is insufficient knowledge for lateral compartmental $\mathrm{OA}$ in comparison to medial compartment OA. This may raise the question of whether similar findings from different radiographic methods are found in end-stage lateral compartment OA. Better understanding of the radiologic characteristics of end-stage lateral OA will be helpful in diagnosing and managing some patients with advanced disease. The purpose of this retrospective study was to examine the demographic and radiographic features of end-stage lateral compartment knee OA (Kellgren-Lawrence grade 3 or 4). We hypothesized that not all persons with end-stage lateral OA have valgus malalignment and that full extension radiographs may underreport radiographic disease severity.

\section{Materials and methods}

We retrospectively reviewed the records of 133 patients who had undergone primary total knee arthroplasty between June 2008 and August 2010. All subjects had lateral compartment OA based on the following criteria: (1) only lateral compartment involvement, (2) only lateral and patellofemoral involvement, or (3) involvement of the lateral and medial compartments (with or without patellofemoral involvement) but with the lateral involvement more severe than the medial involvement according to the compartment-specific Kellgren-Lawrence grade (KLG) [2, 3].

A detailed retrospective review of the medical records of these patients was conducted to extract all pertinent information on the body mass index and gender. Preoperatively, standing hip-knee-ankle radiographs were taken and the mechanical axis angle (MAA) was measured. MAA is the angle between a line from the center of the femoral head running distally to the mid-condylar point between the cruciate ligaments (femoral mechanical axis) and a line from the center of the tibial plateau extending distally to the center of the tibial plafond (tibial mechanical axis) [4]. The neutral MAA was categorized as $0^{\circ}$ to $2^{\circ}$ of varus. The tibia vara angle was defined as the angle between a line perpendicular to the epiphysis and the anatomical axis of the tibia, which was measured using radiographs of the entire lower limb. The line to the epiphysis was measured perpendicular to the line that connected both ends of the epimetaphy seal junction (Fig. 1) [5-10]. Compartment-specific KLG and JSN were measured at extension and $30^{\circ}$ of knee flexion on weightbearing views. Compartment-specific JSN was measured from the center of the medial/lateral condyle to the center of the medial/lateral tibial plateau [11]. To assess reliability, each evaluation (KLG, MAA, tibia vara angle, and tibial slope angle) was measured by two experienced researchers (BYH, HSA) under the supervision of the coauthor (KAJ, with 10 years' musculoskeletal radiology experience), who were blinded to patients' information using the PACS system (INFINITT Healthcare Co Ltd, Seoul, Korea). The average of the two individual mean values was used.

\section{Statistical analysis}

SPSS (Statistical Package for the Social Sciences, v.12.0, Chicago, IL) was used for statistical analyses. For tibia vara angle and tibial slope angle (continuous data), and JSN, ANOVA was used to analyze differences in variables with differing MAA. For comparing KLG (categorical ordinal data), a chi-square test was employed. Bivariate analysis (Spearman's correlation coefficient for categorical data) was used to determine the correlation between radiologic mismatches and variables. The interobserver reliability in measuring variables (KLG, MAA, tibia vara angle, JSN, and tibial slope angle) was evaluated using the intraclass correlation set at a $95 \%$ confidence interval. A level of significance was set at $p<0.05$.

\section{Results}

There were 119 out of 133 knees from females. Endstage lateral compartment knee OA has varus $(37.6 \%)$, neutral $(22.6 \%)$, and valgus (39.8\%) MAA on both-leg standing hip-knee-ankle radiographs. KLG at $30^{\circ}$ of knee flexion (fKLG) was grades 3 and 4 in all patients. However, for the KLG at full extension (eKLG), $54 \%$ of all patients had grades 3 and 4 . The others $(46 \%)$ showed grades 1 and 2 , which caused mismatches of KLG between extension and $30^{\circ}$ of knee flexion (radiologic mismatch). Only the MAA had a negative correlation with radiologic mismatch $(r=-0.486, p<0.01)$ (Table 1). With a more valgus MAA, there was less radiologic mismatch.

We observed significant differences in lateral compartment eKLG/eJSN $(2.3 / 2.3 \mathrm{~mm}$ in varus, $2.5 / 1.9 \mathrm{~mm}$ in 


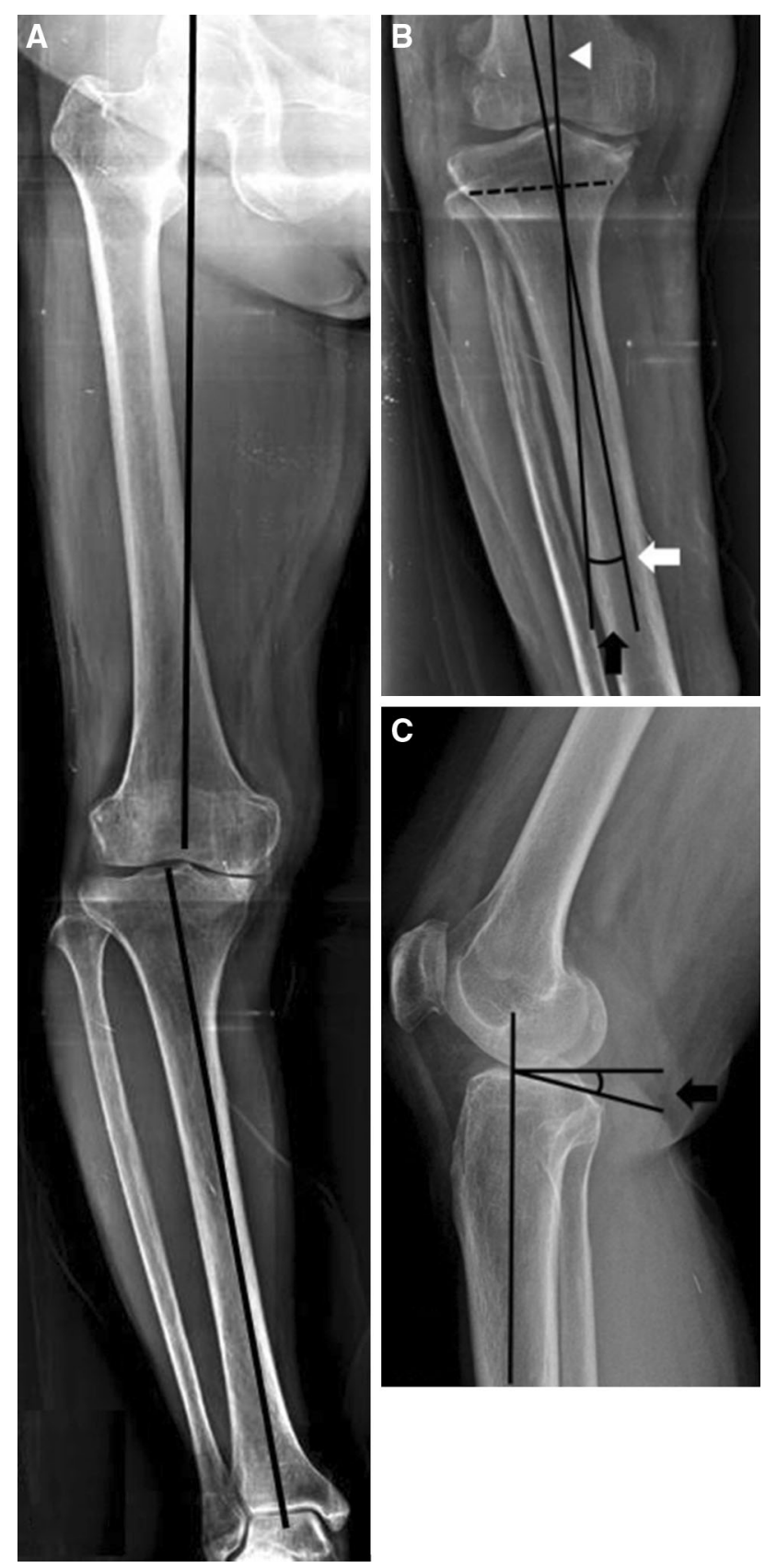

Fig. 1 Mechanical axis angle is the angle between a line from the center of the femoral head to the mid-condylar point between the cruciate ligaments and a line from the center of the tibial plateau to the center of the tibial plafond (a). The tibia vara angle (black arrow) is formed by the line perpendicular to the epiphysis (white arrowhead) and the anatomical axis of the tibia (white arrow) (b). The posterior tibial slope angle is defined as $90^{\circ}$ minus the angle made by the intersection of the line along the longitudinal axis of the tibia and the slope of the medial tibial plateau (c)

neutral, $2.9 / 1.6 \mathrm{~mm}$ in valgus, $p=0.01$ and 0.03 , respectively), tibia vara angle $\left(4.9^{\circ}\right.$ in varus, $4.1^{\circ}$ in neutral, $3.0^{\circ}$ in valgus, $p<0.01$ ), and medial compartment eKLG/eJSN $(2.1 / 3.1 \mathrm{~mm}$ in varus, $2.0 / 3.4 \mathrm{~mm}$ in neutral, $1.8 / 4.3 \mathrm{~mm}$ in valgus, $p<0.01$ and 0.01 , respectively) between MAA groups except for the tibial slope angle $\left(9.7^{\circ}\right.$ in varus, $10.1^{\circ}$ in neutral, $9.8^{\circ}$ in valgus, $p=0.31$ ) (Table 2). The posthoc test showed increased mean lateral compartment eKLG/eJSN in valgus MAA compared to the others, increased tibia vara angle in varus MAA compared to valgus, increased medial compartment eKLG/eJSN in varus, and neutral MAA compared to valgus.

Among all patients, 20 patients $(15.0 \%)$ had bilateral severe lateral OA, and 47 patients $(35.3 \%)$ had contralateral severe medial OA who underwent TKA. There was no difference in BMI or sex between the two groups. However, in the former group, significant older age and increased mismatch was observed.

The intraclass correlation coefficient for inter-tester reliability of KLG, MAA, tibia vara angle, joint space width (JSW), and tibial slope angle was $0.785,0.833$, $0.802,0.753$, and 0.812 , respectively.

\section{Discussion}

This study focused on the radiologic and demographic features of end-stage lateral knee OA. In this study, all patients showed grades 3 and 4 KLGs in their flexion view, representing bone to bone contact (end-stage). However, not all patients showed grades 3 and 4 KLGs on extension views. Valgus and neutral MAA accounted for the majority of our sample with end-stage lateral knee OA. Varus alignment was also paradoxically shown in approximately one-third of those with end-stage lateral knee OA.

Radiographic protocols of the knee in flexion have been shown to improve the detection of JSN by providing better exposure of the location of the greater cartilage changes in the posterior area of the femoral condyles [11-14]. The flexion weight-bearing radiograph is commonly used and is reportedly markedly better than the conventional radiograph in evaluating detection of JSN and disease severity $[15,16]$. The contact zones of femorotibial articulation shift in both area and location as flexion occurs. As the knee is flexed during the stance phase of gait, the femorotibial contact area moves posteriorly and decreases in size. With greater loads per unit of area, the cartilage is more susceptible to degeneration in the contact zones of flexion. Because of this, the sensitivity and specificity of the flexion weight-bearing radiograph is markedly better than the conventional extension radiograph $[15,16]$. In other words, the extension view risks underestimation in diagnosing OA compared to the flexion view.

Despite the generalized loss of articular cartilage in endstage osteoarthritis, JSN is not always found to be consistent between extension and flexion weight-bearing views, contributing to radiologic mismatch. Our study showed that 
Table 1 Correlation coefficient between radiologic mismatch and other factors

\begin{tabular}{|c|c|c|}
\hline & Pearson's correlation coefficient & $p$ value \\
\hline Age, years & 0.096 & 0.29 \\
\hline Sex, M/F & 0.014 & 0.88 \\
\hline Body mass index, $\mathrm{kg} / \mathrm{m}^{2}$ & -0.058 & 0.58 \\
\hline Mechanical axis angle, ${ }^{\circ}$ & -0.486 & $<0.01$ \\
\hline Tibia vara angle, ${ }^{\circ}$ & 0.196 & 0.12 \\
\hline Tibial slope angle, $^{\circ}$ & 0.109 & 0.23 \\
\hline Lateral compartment joint space width at extension, $\mathrm{mm}$ & 0.286 & $<0.01$ \\
\hline Medial compartment joint space width at extension, $\mathrm{mm}$ & -0.129 & 0.14 \\
\hline Lateral compartment joint space width at flexion, $\mathrm{mm}$ & 0.002 & 0.98 \\
\hline Medial compartment joint space width at extension, mm & 0.152 & 0.08 \\
\hline
\end{tabular}

Table 2 Demographic and radiologic features of end-stage lateral knee osteoarthritis with differing alignment

\begin{tabular}{|c|c|c|c|c|}
\hline & Varus $(n=50)$ & Neutral $(n=30)$ & Valgus $(n=53)$ & $p$ value \\
\hline Mean age (range), years & $69.3(57-80)$ & $69.3(60-79)$ & $68.1(57-83)$ & 0.60 \\
\hline Sex, M/F & $4 / 46$ & $3 / 27$ & $7 / 46$ & 0.62 \\
\hline Body mass index, $\mathrm{kg} / \mathrm{m}^{2}$ & $25.1 \pm 3.3$ & $26.2 \pm 2.8$ & $25.8 \pm 3.2$ & 0.44 \\
\hline Lateral compartment Kellgren-Lawrence grade at extension & $2.3 \pm 0.7$ & $2.5 \pm 0.8$ & $2.9 \pm 0.8$ & $<0.01$ \\
\hline Lateral compartment joint space width at extension, $\mathrm{mm}$ & $2.3 \pm 0.8$ & $1.9 \pm 0.9$ & $1.6 \pm 0.8$ & 0.03 \\
\hline Radiologic mismatch, $\%$ & 50.0 & 60.0 & 84.9 & $<0.01$ \\
\hline Medial compartment Kellgren-Lawrence grade at extension & $2.1 \pm 0.4$ & $2.0 \pm 0.4$ & $1.8 \pm 0.5$ & 0.03 \\
\hline Medial compartment joint space width at extension, mm & $3.1 \pm 1.2$ & $3.4 \pm 1.0$ & $4.3 \pm 0.9$ & 0.01 \\
\hline Tibia vara angle, $^{\circ}$ & $4.6 \pm 2.7$ & $3.8 \pm 2.3$ & $2.7 \pm 2.9$ & $<0.01$ \\
\hline Tibial slope angle, ${ }^{\circ}$ & $9.7 \pm 3.1$ & $10.1 \pm 2.8$ & $9.8 \pm 3.2$ & 0.26 \\
\hline
\end{tabular}

$46 \%$ of patients with end-stage lateral OA showed this phenomenon.

Meniscal damage also has an important role in OA. The vast majority of meniscal tears occur in their posterior half and thus chondral damage and loss of joint space occurs when the knee is loaded in the flexed position. Anteriorly, the meniscus and articular cartilage is usually intact, and so in the extended position there is less loss of joint space. Due to a stance phase knee adduction moment, even during normal gait in healthy knees, more load passes through the medial tibiofemoral compartment than through the lateral compartment $[17,18]$. For this reason the extension view is more suited for observing medial compartment OA than lateral disease. However, limb alignment becomes more valgus angulated with an increase in flexion rather than extension [19], which distributes more weight in the lateral compartment and shows lateral JSN in knee flexion.

Previous reports noted that lower extremity malalignment increases the rate of progression of knee osteoarthritis [20-22]. An increase in the varus angle was associated with a significantly increased adjusted risk of having severe medial disease. Also, valgus alignment increases the risk of progression of lateral disease, and an increasing valgus angle is associated with more severe progression of lateral disease [2, 20, 21]. However, in our study, a large proportion of patients with end-stage lateral OA showed varus and neutral alignment. Indeed 'varus' alignment was shown in approximately one-third of patients with endstage lateral knee OA on both-leg standing hip-knee-ankle radiographs. However, it is clear that lateral cartilage loss is advanced by 'valgus' alignment during walking. The knee which is originally valgus is simply seen to be 'varus' on both-leg standing hip-knee-ankle radiographs, but it is not 'varus'. Brouwer et al. [22] reported the prevalence of malalignment in knees without OA in 2290 knees, and observed $25 \%$ with varus alignment, and $36 \%$ with valgus alignment. Interestingly, our study showed that in endstage lateral OA, the distribution of valgus malalignment was similar to the normal knees proportion.

Both knees demonstrated end-stage lateral OA (knock knees) in $15 \%$ of all patients. Of enrolled patients who showed contralateral medial OA, 35.3\% underwent TKA (windblown knees). The patients with contralateral medial compartment OA all had varus MAA in the contralateral 
lower limb. There was no difference in BMI or sex between knock and windblown knees. The former group showed older age than the latter. This suggested that varus alignment would affect the progression of medial compartment OA more, compared to the valgus alignment effect on lateral compartment OA. Brouwer et al. [22] observed a borderline effect of valgus malalignment on the risk of incident OA, while varus malalignment had a larger effect.

Some reported that limb alignment modulates the effect of standard risk factors for progression of OA of the knee, including obesity, quadriceps strength, laxity, and stage of disease [23-25]. In medial compartment OA, limb alignment has a great effect on OA prevalence. However, in lateral $\mathrm{OA}$, another factor beside limb malalignment would have more effect on the lateral compartment than the medial compartment.

This study did have some limitations. First, we performed only a cross-sectional observational study. Second, we attempted to control for this by grouping grades 3 and 4 together (severe disease) and grades 1 and 2 together (mild to moderate disease). In this way, differences between severe and mild to moderate disease are much more likely to indicate a real change in radiographic evidence of disease progression than would have been the case if each of the four grades had been considered separately. Third, enrolled patients in this study were all symptomatic. The symptoms of OA of the knee are typically described as mechanical-that is, they occur with physical activity. Fourth, this study was based on X-ray findings, not on MRI. JSW is determined not only by cartilage thickness, but also other factors such as knee angle, direction of the $\mathrm{X}$-ray beam, and meniscal status. We thought that further study based on MRI would enable assessment of the articular cartilage without being affected by any of these, and could be helpful in uncovering the reason why such mismatch occurs.

Valgus and neutral MAA accounted for the majority of our sample with end-stage lateral knee OA on the both-leg standing hip-knee-ankle radiographs. Varus alignment was also paradoxically shown in approximately one-third of those with end-stage lateral knee OA. Radiographs taken in full extension underreported the degree of OA radiographic severity, with more mismatch being evident with more varus alignment. Varus MAA showed positive correlations with increased tibial vara angle and medial compartment eKLG in end-stage lateral OA.

\section{Conflict of interest None.}

Ethical standards (1) All the patients gave informed consent prior being included in the study; (2) the study was authorized by the local ethical committee and was performed in accordance with the ethical standards of the 1964 Declaration of Helsinki as revised in 2000.
Open Access This article is distributed under the terms of the Creative Commons Attribution 4.0 International License (http:// creativecommons.org/licenses/by/4.0/), which permits unrestricted use, distribution, and reproduction in any medium, provided you give appropriate credit to the original author(s) and the source, provide a link to the Creative Commons license, and indicate if changes were made.

\section{References}

1. Hunter DJ (2009) Insights from imaging on the epidemiology and pathophysiology of osteoarthritis. Radiol Clin North Am 47(4):539-551

2. Khan FA, Koff MF, Noiseux NO, Bernhardt KA, OByrne MM, Larson DR et al (2008) Effect of local alignment on compartmental patterns of knee osteoarthritis. J Bone Joint Surg Am 90(9):1961-1969

3. Kellgren JH, Lawrence JS (1957) Radiological assessment of osteo-arthrosis. Ann Rheum Dis 16(4):494-502

4. Cooke TD, Li J, Scudamore RA (1994) Radiographic assessment of bony contributions to knee deformity. Orthop Clin North Am 25(3):387-393

5. Cooke TD, Sled EA, Scudamore RA (2007) Frontal plane knee alignment: a call for standardized measurement. J Rheumatol 34(9):1796-1801

6. Park IS, Ong A, Nam CH, Ahn NK, Ahn HS, Lee SC, Jung KA (2014) Transepicondylar axes for femoral component rotation might produce flexion asymmetry during total knee arthroplasty in knees with proximal tibia vara. Knee 21(2):369-373

7. Massin P, Gournay A (2011) Optimization of the posterior condylar offset, tibial slope, and condylar roll-back in total knee arthroplasty. J Arthroplasty 26:124-130

8. Utzschneider S, Goettinger M, Weber P, Horng A, Glaser C, Jansson V et al (2011) Development and validation of a new method for the radiologic measurement of the tibial slope. Knee Surg Sports Traumatol Arthrosc 19(10):1643-1648

9. Brandon ML, Haynes PT, Bonamo JR, Flynn MI, Barrett GR, Sherman MF (2006) The association between posterior-inferior tibial slope and anterior cruciate ligament insufficiency. Arthroscopy 22:894-899

10. Yoo JH, Chang CB, Shin KS, Seong SC, Kim TK (2008) Anatomical references to assess the posterior tibial slope in total knee arthroplasty: a comparison of 5 anatomical axes. J Arthroplasty 23:586-592

11. Shelbourne KD, Dickens JF (2006) Digital radiographic evaluation of medial joint space narrowing after partial meniscectomy of bucket-handle medial meniscus tears in anterior cruciate ligament-intact knees. Am J Sports Med 34(10):1648-1655

12. Merle-Vincent F, Vignon E, Brandt K, Piperno M, Coury-Lucas F, Conrozier T et al (2007) Superiority of the Lyon schuss view over the standing anteroposterior view for detecting joint space narrowing, especially in the lateral tibiofemoral compartment, in early knee osteoarthritis. Ann Rheum Dis 66(6):747-753

13. Rosenberg TD, Paulos LE, Parker RD, Coward DB, Scott SM (1988) The forty-five-degree posteroanterior flexion weightbearing radiograph of the knee. J Bone Joint Surg Am 70(10):1479-1483

14. Piperno M (1998) Hellio Le Graverand MP, Conrozier T, Bochu M, Mathieu P, Vignon E. Quantitative evaluation of joint space width in femorotibial osteoarthritis: comparison of three radiographic views. Osteoarthr Cartil 6(4):252-259

15. Maquet PG, Berg AJ, Van de Simonet JC (1975) Femorotibial weight-bearing areas. Experimental determination. J Bone Joint Surg Am 57(6):766-771 
16. Kurosawa H, Fukubayashi T, Nakajima H (1980) Load-bearing mode of the knee joint: physical behavior of the knee joint with or without menisci. Clin Orthop Relat Res 149:283-290

17. Morrison JB (1970) The mechanics of the knee joint in relation to normal walking. J Biomech 3(1):51-61

18. Andriacchi TP (1994) Dynamics of knee malalignment. Orthop Clin North Am 25(3):395-403

19. Koshino T, Takeyama M, Jiang LS, Yoshida T, Saito T (2002) Underestimation of varus angulation in knees with flexion deformity. Knee 9(4):275-279

20. Sharma L, Song J, Felson DT, Cahue S, Shamiyeh E, Dunlop DD (2001) The role of knee alignment in disease progression and functional decline in knee osteoarthritis. JAMA 286(2):188-195

21. Cicuttini F, Wluka A, Hankin J, Wang Y (2004) Longitudinal study of the relationship between knee angle and tibiofemoral cartilage volume in subjects with knee osteoarthritis. Rheumatology (Oxford) 43(3):321-324
22. Brouwer GM, van Tol AW, Bergink AP, Belo JN, Bernsen RM, Reijman M et al (2007) Association between valgus and varus alignment and the development and progression of radiographic osteoarthritis of the knee. Arthritis Rheum 56(4):1204-1211

23. Felson DT, Goggins J, Niu J, Zhang Y, Hunter DJ (2004) The effect of body weight on progression of knee osteoarthritis is dependent on alignment. Arthritis Rheum 50(12):3904-3909

24. Sharma L, Lou C, Cahue S, Dunlop DD (2000) The mechanism of the effect of obesity in knee osteoarthritis: the mediating role of malalignment. Arthritis Rheum 43(3):568-575

25. Cerejo R, Dunlop DD, Cahue S, Channin D, Song J, Sharma L (2002) The influence of alignment on risk of knee osteoarthritis progression according to baseline stage of disease. Arthritis Rheum 46(10):2632-2636 